\title{
All-optical switching in a two-channel waveguide with cubic-quintic nonlinearity
}

\author{
Rodislav Driben ${ }^{1}$, Boris A. Malomed ${ }^{1}$, and Pak L. Chu ${ }^{2}$
}

\begin{abstract}
We consider dynamics of spatial beams in a dual-channel waveguide with competing cubic and quintic (CQ) nonlinearities. Gradually increasing the power in the input channel, we identify four different regimes of the pulse's coupling into the cross channel, which alternate three times between full pass and full stop, thus suggesting three realizations of switching between the channels. As in the case of the Kerr (solely cubic) nonlinearity, the first two regimes are the linear one, and one dominated by the self-focusing nonlinearity, with the beam which, respectively, periodically couples between the channels, or stays in the input channel. Further increase of the power reveals two novel transmission regimes, one characterized by balance between the competing nonlinearities, which again allows full coupling between the channels, and a final regime dominated by the selfdefocusing quintic nonlinearity. In the latter case, the situation resembles that known for a selfrepulsive Bose-Einstein condensate trapped in a double-well potential, which is characterized by strong symmetry breaking; accordingly, the beam again abides in the input channel, contrary to an intuitive expectation that the self-defocusing nonlinearity would push it into the cross channel. The numerical results are qualitatively explained by a simple analytical model based on the variational approximation.
\end{abstract}

\section{INTRODUCTION AND THE MODEL}

Dynamics of spatial light beams in nonlinear planar waveguides with a multi-channel built-in structure is a subject of fundamental interest to nonlinear optics, and has vast potential for applications to all-optical routing of data streams. The basic model of the multi-channel system amounts to the nonlinear Schrödinger (NLS) equation for the local amplitude $\Psi(z, x)$ of the electromagnetic wave, where $z$ and $x$ are the propagation and transverse coordinates in the waveguide. In a normalized form, the NLS equation is [1]

$$
i \Psi_{z}+\Psi_{x x}-U(x) \Psi+\delta n\left(|\Psi|^{2}\right) \Psi=0,
$$

where the second term accounts for the diffraction of light in the paraxial approximation, $U(x)$ is the profile of the transverse modulation of the refractive index that accounts for the multi-channel structure, and $\delta n\left(|\Psi|^{2}\right)$ is a nonlinear correction to the refractive index. In the case of the ordinary self-focusing (SF) Kerr nonlinearity, $\delta n\left(|\Psi|^{2}\right)=n_{2}|\Psi|^{2}$, with $n_{2}>0$.

The interplay of the diffraction and SF nonlinearity gives rise to channel-trapped spatial solitons. In the model with the Kerr nonlinear term and harmonic transverse modulation, $U(x)=U_{0} \cos (2 \pi x / L)$, trapped solitons and a possibility of their switching were studied in Ref. [1]. It was found that the soliton's integral power, $P=\int_{-\infty}^{+\infty}|\Psi(x)|^{2} d x$, which is a dynamical invariant of the NLS equation, may take any value, $0<P<\infty$, the entire solution family being stable. Switching, i.e., transfer of the soliton into an adjacent channel, was demonstrated under the action of a "hot spot" modeled by an extra term $\sim \delta\left(x-x_{0}\right) \delta\left(z-z_{0}\right) \Psi$ in the equation, with $x_{0}$ fixed at the midpoint between the channels.

The same model as in Ref. [1], with $z$ replaced by time $t$ (but without the hot-spot term) was later introduced for an effectively one-dimensional Bose-Einstein condensate (BEC) with attraction between atoms, loaded in an optical-lattice potential 2]. Spatial solitons in a model of the Kerr-nonlinear optical waveguide with the periodic transverse modulation in the form of the Kronig-Penney profile (a periodic array of rectangular troughs, see below) were considered in Refs. [3]. In optics, a system of parallel guiding cores can be created not only through a small periodic variation of the material's refractive index, but also in a virtual form, by means of a system of strong transverse laser beams illuminating a sample of a photorefractive material in the ordinary polarization, while the signal beam is launched in the extraordinary polarization [4].

The functionality of optical beam-handling schemes may be greatly enhanced in media with competing nonlinearities, the simplest example of which is provided by a combination of the SF cubic and self-defocusing (SDF) quintic terms. Observations of the cubic-quintic (CQ) optical nonlinearity were reported in the PTS crystal [5] , chalcogenide glasses [6] and some other materials [7]. In fact, the CQ nonlinearity occurs due to an intrinsic nonlinear resonance in the material, which also gives rise to strong two-photon absorption 8 ; ; however, experiments with spatial solitons require short propagation distances, on the order of a few centimeters [4, 9], over which effects of the loss may be insignificant [10].

The NLS equation with the CQ nonlinearity and the multi-channel guiding structure can be rescaled into the following form [11]:

$$
i \Psi_{z}+\Psi_{x x}=U(x) \Psi-2|\Psi|^{2} \Psi+|\Psi|^{4} \Psi
$$


In the uniform medium $[U(x)=0]$, Eq. (2) gives rise to a well-known family of stable solitons [12],

$$
\Psi(x, z)=\exp (i k z) \sqrt{\frac{2 k}{1+\sqrt{1-4 k / 3} \cosh (2 \sqrt{k} x)}},
$$

with the propagation constant $k$ taking values in a finite interval, $0<k<3 / 4$. In Ref. [11], solitons were studied in CQ model (2) with a single guiding channel of a rectangular shape. A remarkable feature of the channel-trapped CQ solitons is bistability of the guided-soliton family: in the same region as mentioned above, $0<k<3 / 4$, the channel supports a single soliton state, but the full existence region extends to an additional interval, $3 / 4<k<k_{\max }$, where two different solitons are found for each $k$, both being stable (the soliton bistability does not occur in the same guiding channel if the model contains only the cubic nonlinearity [13]). The CQ nonlinearity was combined with the multi-channel structure of the Kronig-Penney type in Ref. [14]. In addition to fundamental solitons, many families of higher-order solitons, also featuring the bistability, were found in the latter model.

For applications to all-optical switching, more relevant solutions are not static solitons, but rather dynamical states featuring periodic transfer (coupling) of the beam between two adjacent guiding cores. In this context, the most appropriate model is one with two channels, similar to nonlinear directional couplers operating in the temporal domain, which are based on dual-core fibers (see Refs. 15] and references therein). The objective of the present work is to report numerical results and some analytical approximations for the beam switching in the dual-channel $\mathrm{CQ}$ model, which is based on Eq. (2) with

$$
U(x)= \begin{cases}0, & |x|<\frac{1}{2} L \text { and }|x|>\frac{1}{2} L+D, \\ -U_{0}, & \frac{L}{2}<|x|<\frac{1}{2} L+D\end{cases}
$$

where $D, U_{0}$ and $L$ are, respectively, the width and depth of each channel, and the thickness of the buffer layer between them. A fundamental difference from earlier studied two-channel models with the Kerr nonlinearity is expected dependence of the coupling on the beam's input power $P$ : in the Kerr model, the increase of $P$ leads simply to transition from the nearly-linear-coupling regime to a nonlinear one, in which the beam stays in the input channel (coupling suppression). In the CQ model, a more sophisticated dependence on $P$ may be anticipated, due to the competition between the cubic SF and quintic SDF terms. The main qualitative result demonstrated below is that the coupling suppression is changed by restoration of the coupling at larger values of $P$ (at which the SF and SDF terms are in balance), and finally again by suppression of the coupling at very large $P$, when the quintic term dominates, even if the suppression of coupling by the self-defocusing nonlinearity is a counterintuitive effect. In Section III we display typical examples of the four distinct transmission modes, and then present a diagram which provides for overall description of the switching in the dual-channel waveguide with the CQ nonlinearity. Using values of strengths of the cubic and quintic SF and SDF nonlinearities in available materials, we estimate that the switching can be achieved at power densities of the input beam on the order of $\mathrm{GW} / \mathrm{cm}^{2}$, the corresponding coupling distance being $\lesssim 1 \mathrm{~cm}$.

In addition to the numerical results, in Section III] we report results of a simple analytical approximation that provide for a qualitative explanation of the switchings in the present model. The analytical approach is based on a variational approximation, similar to that developed earlier in detail for temporal signals (solitons) in dual-core fibers [15, 16, 17] (see also Chapter 6 of review [18]).

\section{COUPLING DYNAMICS AND SWITCHING DIAGRAMS}

To simulate the transmission of the spatial beam in the system described by Eq. (2), we integrated this equation with initial conditions (at $z=0$ ) corresponding to a Gaussian beam, with amplitude $A$ and width $W$, launched into one channel:

$$
\Psi_{0}=A \exp \left[-\frac{1}{W^{2}}\left(x-\frac{L+D}{2}\right)^{2}\right]
$$

$[x=(L+D) / 2$ is the center of the first channel as per Eq. (44)]. The splitting of light between the two channels is quantified by respective integral powers,

$$
P_{1}=\int_{L / 2}^{(L+D) / 2}|\Psi|^{2} d x, P_{2}=\int_{-(L+D) / 2}^{-L / 2}|\Psi|^{2} d x .
$$


Equation (2) was solved numerically by means of the split-step method. The input power was gradually increased, and the evolution of the power distribution across the waveguide was monitored in the course of the simulations. Collecting numerical results, it was found that they are adequately represented by fixing the parameters at $L=D=U_{0}=W=2$. This implies that the width of the channels is equal to the thickness of the buffer layer between them, and the diffraction and channel-trapping terms are comparable to the nonlinear ones when they are in balance. Results for other values of the parameters will be displayed too.

If the input power is small, nonlinear terms in Eq. (2) are negligible in comparison to the diffraction, which leads to the ordinary picture of the periodic coupling of power between the channels, as shown in Fig. 1. Note that the transmission length equal to coupling length $Z_{\text {coupl }}$, i.e., half-period of the coupling (as in Fig. 1), provides for the full transfer of the beam into the cross channel.

Increase of the input power makes the SF cubic nonlinearity dominant, switching (as usual) the transmission mode from the periodic coupling to one with the power abiding in the input channel. This situation is illustration by Fig. 2 .

The two transmission modes shown in Figs. 1 and 2, and the switching between them are qualitatively the same as in ordinary dual-core models with the Kerr nonlinearity [15. Switching to an essentially new transmission regime, which is specific to the CQ medium, occurs with further increase of the power, in a range where the SF cubic and SDF quintic terms balance each other, rendering the situation similar to that in the linear model. This new quasi-linear regime is illustrated by Fig. 3. It is noteworthy that the linear-coupling mode in Fig. 1, and its strongly nonlinear but similar counterpart in Fig. 3 have nearly equal coupling lengths: for equal values of the system's parameters and beam's width, $Z_{\text {coupl }}^{\text {(nonlin) }} / Z_{\text {coupl }}^{\text {(lin) }} \approx 7 / 6$.

Extra (final) switching occurs, if one keeps increasing the input power, to a transmission regime in which the SDF quintic term dominates. "Naively", one might expect that SDF nonlinearity would only enhance coupling between the guiding cores; however, it actually suppresses the coupling, as shown in a typical example in Fig. 4. As observed in the figure, this regime is not completely equivalent to one in which the coupling was suppressed by the dominant SF cubic term (cf. Fig. 2), as the present transmission mode features conspicuous oscillations; nevertheless, the coupling is largely suppressed in this case too.

A result similar to that demonstrated in Fig. 4 is known in a model of a Bose-Einstein condensate (BEC) with repulsion between atoms (which is formally tantamount to SDF cubic nonlinearity) trapped in a double-well potential, the two potential minima playing the same role as the dual-channel configuration. The corresponding Gross-Pitaevskii equation (the counterpart of the NLS equation for the BEC) gives rise to stable asymmetric states (see Ref 19] and references therein). This symmetry-breaking effect has also been observed in experiment [20] [in Ref. 21], a similar effect was demonstrated, theoretically and experimentally (using a photorefractive crystal), in a medium with saturable nonlinearity].

Results of systematic simulations are summarized in the form of a switching diagram, which is displayed in Fig. 5. To measure the coupling rate, we define $\varepsilon \equiv\left(P_{2}\right)_{\max } / P$, as the maximum share of the total input power $(P)$, which is coupled into the second channel. The diagram plots $\varepsilon$ versus the total power itself; to demonstrate the generic character of the dependence, it is presented for three different values of the width of the input pulse (5) (including $W=2$, for which examples of the transmission modes are displayed in Figs. 1-4). The regimes with $\varepsilon$ approaching 1 , and with $\varepsilon \ll 1$ are, respectively, those featuring nearly complete periodic transfer of the power between the channels, and suppression of the coupling. In accordance with what was displayed above by dint of examples, the strong coupling takes place at small $P$, which corresponds to the nearly linear transmission, and in a relatively narrow interval of $P$ providing for the balance between the SF and SDF nonlinearities. In two broad intervals, where either the cubic term or its quintic competitor dominate, the coupling is suppressed. For applications, a beneficial feature of the $\varepsilon(P)$ dependences in Fig. 5 is the steep transition between regimes of the two types, i.e., sharp switching. Figure 5 also suggests that exact values of the power at which the switchings take place can be tuned by adjusting the width of the input pulse.

To conclude this section, we note that, with typical values of the cubic and quintic nonlinear coefficients for chalcogenide glasses reported in Refs. [8], $n_{2}=2.2 \times 10^{-4} \mathrm{~cm}^{2} / \mathrm{GW}$, and $n_{4}=-7.9 \times 10^{-5} \mathrm{~cm}^{4} /(\mathrm{GW})^{2}$ (they are defined so that the total nonlinear correction to the refractive index is $\delta n=n_{2} I+n_{4} I^{2}$, where $I$ is the power density in the optical beam), the switchings specific to the CQ medium are attained in a ballpark of $I=3 \mathrm{GW} / \mathrm{cm}^{2}$, which provides for the balance between the SF and SDF effects, and is achievable in the experiment [6]. Analysis of the corresponding wave-propagation equation written in physical units demonstrates that the respective coupling length (in the quasi-linear regime of balanced SF and SDF) may take values in the range of 0.1 to $1 \mathrm{~cm}$. 


\section{ANALYTICAL APPROXIMATION}

The underlying equation (2) can be derived from the Lagrangian

$$
L=\int_{-\infty}^{+\infty}\left[\frac{i}{2}\left(\Psi^{*} \Psi_{z}-\Psi_{z}^{*} \Psi\right)-\left|\Psi_{x}\right|^{2}-U(x)|\Psi|^{2}+|\Psi|^{4}-\frac{1}{3}|\Psi|^{6}\right] d x .
$$

To apply the variational approximation to the present model, we assume a situation with very narrow and deep channels, separated by a broad buffer layer. The field configuration is then approximated as follows: inside each channel, i.e., in regions $|x-(L \pm D) / 2|<D / 2$ [see Eq. (4)],

$$
\Psi(x, z)=A_{ \pm}(z) \cos \left(\frac{x-(L \pm D) / 2}{D}\right)
$$

with complex amplitudes $A_{ \pm}$(this ansatz emulates the ground state of a quantum-mechanical particle in an infinitely deep potential box, therefore it vanishes at edges of the channel). The form of the ansatz outside the channels is also borrowed from quantum mechanics, emulating a superposition of exponentially decaying ground-state wave functions behind the edges of very deep but finite potential boxes,

$$
\Psi(x, z)=\sum_{+,-} A_{ \pm}(z) \exp \left(-\sqrt{U_{0}-(\pi / D)^{2}}\left|x-\frac{L \pm D}{2}\right|\right) .
$$

The substitution of the inner and outer parts of the ansatz, (8) and (9), in Lagrangian (7) and integration lead to the following effective Lagrangian:

$$
\begin{aligned}
\frac{4}{D} L_{\mathrm{eff}}= & \sum_{+,-}\left\{i\left[A_{ \pm}^{*} A_{ \pm}^{\prime}-A_{ \pm}\left(A_{ \pm}^{*}\right)^{\prime}\right]+2\left[U_{0}-\left(\frac{\pi}{D}\right)^{2}\right]\left|A_{ \pm}\right|^{2}+\frac{3}{2}\left|A_{ \pm}\right|^{4}-\frac{5}{12}\left|A_{ \pm}\right|^{6}\right\} \\
& +\frac{4}{D} \sqrt{U_{0}-\left(\frac{\pi}{D}\right)^{2}} \exp \left(-\sqrt{U_{0}-\left(\frac{\pi}{D}\right)^{2}}(L+D)\right)\left(A_{+} A_{-}^{*}+A_{-} A_{+}^{*}\right)
\end{aligned}
$$

where the prime stands for $d / d z$. Note that the last term in (10), which accounts for the interaction between the beams trapped in the two channels, was calculated by means of a general method elaborated in Ref. 22] (see also section 2.3 in review [18]). This method only assumes that, in a vicinity of each channel, the tail created by the other beam is much weaker than the field of the beam trapped in the given channel (note that $L+D$ is the distance between centers of the two channels).

The Euler-Lagrange equations, $\partial L_{\mathrm{eff}} / \partial A_{ \pm}^{*}-\left[\partial L_{\mathrm{eff}} / \partial\left(A_{ \pm}^{*}\right)^{\prime}\right]^{\prime}=0$ can be cast in a real form, by defining $A_{ \pm} \equiv$ $R_{ \pm} e^{i \phi_{ \pm}}$, where $R_{ \pm}$and $\phi_{ \pm}$are real amplitudes and phases:

$$
\begin{aligned}
R_{+}^{\prime} & =\kappa R_{-} \sin \phi, R_{-}^{\prime}=-\kappa R_{+} \sin \phi, \\
\phi_{ \pm}^{\prime} & =\left[-\frac{3}{2}+\frac{5}{8}\left(R_{+}^{2}+R_{-}^{2}\right)\right]\left(R_{\mp}\right)^{2}+\kappa(\cos \phi) \frac{R_{\mp}}{R_{ \pm}},
\end{aligned}
$$

where the effective constant of the tunnel coupling between the two guiding cores is

$$
\kappa \equiv \frac{4}{D} \sqrt{U_{0}-\left(\frac{\pi}{D}\right)^{2}} \exp \left(-\sqrt{U_{0}-\left(\frac{\pi}{D}\right)^{2}}(L+D)\right) .
$$

An obvious consequence of Eqs. (11) and (12) is the conservation of $P \equiv R_{+}^{2}+R_{-}^{2}$ (it is proportional to the total power of the beam), and the fact that an equation for $\phi_{+}+\phi_{-}$is detached from the other equations. Thus, defining

$$
\begin{gathered}
R_{+} \equiv \sqrt{P} \cos (\theta / 2), R_{-} \equiv \sqrt{P} \sin (\theta / 2), \phi \equiv \phi_{+}-\phi_{-}, \\
\lambda \equiv(P / 8)(12-5 P),
\end{gathered}
$$


we end up with a dynamical system

$$
\begin{gathered}
\theta^{\prime}=-\kappa \sin \phi, \\
\phi^{\prime}=\lambda \cos \theta-\kappa(\cos \phi) \cot \theta,
\end{gathered}
$$

which conserves the Hamiltonian,

$$
H=\left(\kappa \cos \phi-\frac{1}{2} \lambda \sin \theta\right) \sin \theta
$$

Dynamical system (16) is similar to that which was derived, in the simplest version of the variational approximation, for a two-component temporal soliton in a dual-core optical fiber with the Kerr nonlinearity [16, 17]. The only significant difference is that the present CQ model gives rise to nonlinearity coefficient $\lambda$ which changes its sign with the increase of the beam's power $P$, see Eq. (15) [ $\kappa$ is always positive, according to its definition (13)].

In the framework of this approximation, solutions corresponding to various coupling modes found above by means of numerical simulations of Eq. (2) with initial condition (5) are obtained from the initial condition with the beam launched into one channel, i.e., $\theta=0$ or $\theta=\pi$, according to Eqs. (14). As seen from Eq. (17), this initial condition yields $H=0$, hence the dynamical trajectory in the $(\theta, \phi)$ plane, $H=$ const, is generated by the equation

$$
\cos \phi=\frac{\lambda}{2 \kappa} \sin \theta
$$

It follows from Eq. (18) that the trajectories take completely different forms in cases $|\lambda|<2 \kappa$ and $|\lambda|>2 \kappa$, see examples shown in Figs. 6(a) and (b). The former case corresponds to weak effective nonlinearity, i.e., to the coupling mode with either small input power, or with balanced SF and SDF terms, cf. Figs. 1 and 3, respectively. In qualitative agreement with the results of numerical simulations of Eq. (2), in this (quasi-linear) case the trajectories run along the $\theta$ direction, which means periodic transfer of power between the channels. Note that Eq. (15) makes it possible to predict intervals of power $P$ in which the quasi-linear coupling occurs: $P<(2 / 5)(3-\sqrt{9-20 \kappa})$, and $(2 / 5)(3+\sqrt{9-20 \kappa})<P<(2 / 5)(3+\sqrt{9+20 \kappa})$ [these expressions imply constraint $\kappa<9 / 20$, which complies with the fact that expression (13) yields small $\kappa]$. On the other hand, in the case of strong nonlinearity, $|\lambda|>2 \kappa$, i.e., in intervals $(2 / 5)(3-\sqrt{9-20 \kappa})<P<(2 / 5)(3+\sqrt{9-20 \kappa})$ and $P>(2 / 5)(3+\sqrt{9+20 \kappa})$, Fig. 6(b) displays trajectories confined to a vicinity of $\theta=0$ or $\theta=\pi$, which corresponds to the suppression of coupling, cf. Figs. 2 and 4 . Thus, the variational approximation provides for a qualitatively correct explanation of the numerical results presented above.

\section{CONCLUSION}

We have investigated possibilities of realizing power-controlled switching of spatial light beams in the dual-channel planar waveguide with the cubic-quintic nonlinearity. The interplay of the transverse diffraction and self-focusing (cubic) and defocusing (quintic) nonlinearities gives rise to two transmission modes similar to those known in models with the cubic nonlinearity, i.e., periodic coupling in the nearly linear situation, and suppression of the coupling in the mode dominated by the cubic term, and two additional regimes, one with the periodic coupling restored due to the balance between the competing nonlinearities, and the other (final) transmission mode with the coupling suppressed again when the self-defocusing quintic term dominates. Three switchings (between the four distinct transmission regimes) are not strongly affected by variation of the system's parameters, but they feature sharpness with variation of the power, especially the reverse switching from the coupling-suppressed mode dominated by the cubic nonlinearity to the strong-coupling one provided by the balance between the self-focusing and defocusing nonlinearities. A simple analytical approximation, which reduces the model to a Hamiltonian dynamical system with one degree of freedom, qualitatively explains all these coupling models and switchings between them.

This work was supported, in a part, by a Strategic Research Grant of the City University of Hong Kong (project No.7001705), and by the Israel Science Foundation through a Center-of-Excellence grant No. 8006/03. R.D. and B.A.M. appreciate hospitality of the Optoelectronics Research Centre at the Department of Electronics Engineering, City University of Hong Kong.

[1] B. A. Malomed, Z. H. Wang, P. L. Chu, and G. D. Peng, J. Opt. Soc. Am. B 16, 1197 (1999). 
[2] G. L. Alfimov, V. V. Konotop, and M. Salerno, Europhys. Lett. 58, 7 (2002).

[3] W. Li and A. Smerzi, Phys. Rev. E 70, 016605 (2004); B. T. Seaman, L. D. Carr, and M. J. Holland, Phys. Rev. A 71 , $033622(2005)$.

[4] J. W. Fleischer, M. Segev, N. K. Efremidis, and D. N. Christodoulides, Nature 422, 147 (2003).

[5] B. L. Lawrence, M. Cha, J. U. Kang, W. Torruellas, G. Stegeman, G. Baker, J. Meth, and S. Etemad, Electr. Lett. 30, 889 (1994); E. W. Wright, B. L. Lawrence, W. Torruellas, and G. I. Stegeman, Opt. Lett. 20, 2481 (1995); B. L. Lawrence and G. I. Stegeman, Opt. Lett. 23, 591 (1998).

[6] F. Smektala, C. Quemard, V. Couderc, and A. Barthélémy, J. Non-Cryst. Solids 274, 232 (2000); K. Ogusu, J. Yamasaki, S. Maeda, M. Kitao, and M. Minakata, Opt. Lett. 29, 265 (2004).

[7] C. Zhan, D. Zhang, D. Zhu, D. Wang, Y. Li, D. Li, Z. Lu, L. Zhao, and Y. Nie, J. Opt. Soc. Am. B 19, 369 (2002).

[8] G. Boudebs, S. Cherukulappurath, H. Leblond, J. Troles, F. Smektala, and F. Sanchez, Opt. Commun. 219, 427 (2003); F. Sanchez, G. Boudebs, S. Cherukulappurath, H. Leblond, J. Troles, and F. Smektala, J. Nonlin. Opt. Phys. \& Mat. 13, 7 (2004).

[9] S. Maneuf, and F. Reynaud, Opt. Commun. 65, 325 (1988); J. S. Aitchison, A. M. Weiner, Y. Silberberg, M. K. Oliver, J. L. Jackel, D. E. Leaird, E. M. Vogel, and P. W. E. Smith, Opt. Lett. 15, 471 (1990).

[10] Y.-F. Chen, K. Beckwitt, F. W. Wise, and B. A. Malomed, Phys. Rev. E 70, 046610 (2004).

[11] B. V. Gisin, R. Driben, and B. A. Malomed, J. Opt. B: Quant. Semiclass. Opt. 6, S259 (2004).

[12] Kh. I. Pushkarov, D. I. Pushkarov, and I. V. Tomov, Opt. Quant. Electr. 11, 471 (1979); S. Cowan, R. H. Enns, S. S. Rangnekar, and S. S. Sanghera, Can. J. Phys. 64, 311 (1986).

[13] B. V. Gisin and A. A. Hardy, Phys. Rev. A 48, 3466 (1993).

[14] I. M. Merhasin, B. V. Gisin, R. Driben, and B. A. Malomed, Phys. Rev. E 71, 016613 (2005).

[15] I. M. Uzunov, R. Muschall, M. Gölles, Yu. S. Kivshar, B. A. Malomed, and F. Lederer, Phys. Rev. E 51, 2527 (1995); P. L. Chu, Yu. S. Kivshar, B. A. Malomed, G. D. Peng, and M. L. Quiroga-Teixeiro, J. Opt. Soc. Am. B 12, 898 (1995).

[16] C. Paré and M. Fłorjańczyk, Phys. Rev. A 41, 6287 (1990); A. I. Maimistov, Kvant. Elektr. 18, 758 (1991) [Sov. J. Quant. Electr. 21, 687 (1991)].

[17] P. L. Chu, B. A. Malomed, and G. D. Peng, J. Opt. Soc. B 10, 1379 (1993).

[18] B. A. Malomed, in: Progress in Optics 43, p. 71 (E. Wolf, editor: North Holland, Amsterdam, 2002).

[19] R. K. Jackson and M. Weinstein, J. Stat. Phys. 116, 881 (2004).

[20] M. Albiez, R. Gati, J. Fölling, S. Hunsmann, M. Cristiani, and M. K. Oberthaler, Phys. Rev. Lett. 95, 010402 (2005); R. Gati, M. Albiez, J. Fölling, B. Hemmerling, and M. K. Oberthaler, Appl. Phys. B 82, 207 (2006).

[21] P. G. Kevrekidis, Z. Chen, B. A. Malomed, D. J. Frantzeskakis, and M. I. Weinstein, Phys. Lett. A 340,275 (2005).

[22] B. A. Malomed, Phys. Rev. E 58, 7928 (1998). 


\section{Figure Captions}

Fig. 1. (Color online) The first half-period of the quasi-linear coupling regime, for input beam (5) with $A=0.2$ and $W=2$ : (a) contour plots of the power distribution; (b) integral powers in the channels, defined as per Eq. (6), vs. the propagation distance.

Fig. 2. (Color online) The same as in Fig. 1, but after the switch to the transmission regime dominated by the self-focusing cubic term. In this case, the input amplitude is $A=1$, i.e., the total power is larger by a factor of 25 than in the linear regime displayed in Fig. 1. A longer transmission distance than in Fig. 1 is shown here, to make it sure that the coupling to the the cross channel never commences.

Fig. 3. (Color online) The same as in Fig. 2, but after the reverse switch to the regime in which the self-focusing and defocusing terms (cubic and quintic ones) are in balance, thus again allowing strong coupling between the channels. In this case, the input amplitude in Eq. (5) is $A=\sqrt{2}$, i.e., the total power is twice as large as in the case shown in Fig. 2. Unlike Fig. 1, about 1.5 periods of the coupling is shown here, to demonstrate the stability of the periodic coupling (actually, it remains stable indefinitely long, in more extensive simulations).

Fig. 4. (Color online) The same as Fig. 3, but after the final switch to the transmission mode with suppressed coupling. The amplitude of the input pulse is $A=1.8$, hence the total input power exceeds that in Fig. 3 by a factor of 1.62 .

Fig. 5. (Color online) Switching diagrams, which show the maximum share of the input power coupled into the cross channel, as a function of the input power itself. The diagrams are presented for initial Gaussian beams (5) with three different values of the width, $W=1,2$, and $2 \sqrt{2}$ (recall examples of the four transmission regimes displayed in Figs. 1-4 pertain to $W=2$ ).

Fig. 6. Typical dynamical trajectories in the phase plane $(\theta, \phi)$, as generated by Eq. (18): $(\mathrm{a}) \lambda /(2 \kappa)=0.5$, which corresponds to the quasi-linear coupling mode; (b) $\lambda /(2 \kappa)=2$, corresponding to the coupling-suppressed mode, dominated by the nonlinearity. 
This figure "Fig1a.jpg" is available in "jpg" format from: http://arxiv.org/ps/nlin/0605010v1 
This figure "Fig1b.jpg" is available in "jpg" format from: http://arxiv.org/ps/nlin/0605010v1 
This figure "Fig2a.jpg" is available in "jpg" format from: http://arxiv.org/ps/nlin/0605010v1 
This figure "Fig2b.jpg" is available in "jpg" format from: http://arxiv.org/ps/nlin/0605010v1 
This figure "Fig3a.jpg" is available in "jpg" format from: http://arxiv.org/ps/nlin/0605010v1 
This figure "Fig3b.jpg" is available in "jpg" format from: http://arxiv.org/ps/nlin/0605010v1 
This figure "Fig4a.jpg" is available in "jpg" format from: http://arxiv.org/ps/nlin/0605010v1 
This figure "Fig4b.jpg" is available in "jpg" format from: http://arxiv.org/ps/nlin/0605010v1 
This figure "Fig5.jpg" is available in "jpg" format from: http://arxiv.org/ps/nlin/0605010v1 
This figure "Fig6a.jpg" is available in "jpg" format from: http://arxiv.org/ps/nlin/0605010v1 
This figure "Fig6b.jpg" is available in "jpg" format from: http://arxiv.org/ps/nlin/0605010v1 